CONCISE REPORT

\title{
MMP profile in paired serum and synovial fluid samples of patients with rheumatoid arthritis
}

\author{
I Tchetverikov, H K Ronday, B van El, G H Kiers, N Verziil, J M TeKoppele, T W J Huizinga, \\ J DeGroot, R Hanemaaijer
}

Ann Rheum Dis 2004;63:881-883. doi: 10.1136/ard.2003.013243

Objective: To analyse matrix metalloproteinases (MMPs) and tissue inhibitor-1 of MMPs (TIMP-1) levels in the systemic circulation and synovial fluid (SF) of patients with RA and to compare these levels with inflammatory and collagen degradation markers.

Methods: ProMMP-1, $-2,-3,-8,-9$, TIMP-1, levels of MMP/ $\alpha_{2}$-macroglobulin complexes, and collagen degradation products were measured by sandwich ELISA, activity assays, and HPLC in paired SF and serum samples from 15 patients with RA and 13 with $O A$.

Results: MMPs were higher in SF of patients with RA than in $\mathrm{OA}$ or controls. MMP levels in SF of patients with OA were higher than in controls. In serum, levels of proMMP-3, -8 and -9 were higher in patients with RA than in OA or controls, whereas only proMMP-8 and -9 were higher in serum of patients with $\mathrm{OA}$ than in controls. A strong correlation was seen between serum and SF levels of MMP-8 and -9 in RA. Increased levels of $M M P / \alpha_{2}$-macroglobulin complexes indicated an MMP/TIMP imbalance in serum and SF in RA. SF hydroxyproline correlated significantly with SF levels of proMMP-9 in RA.

Conclusions: Systemic MMP-8 and -9 levels represent the situation in the inflamed joint; MMP-9 is likely to be involved in degradation of joint collagen. The hypothesis of MMP/ TIMP imbalance in RA is strengthened.

M atrix metalloproteinases (MMPs) are a group of $\mathrm{Zn}^{2+}$ dependent extracellular enzymes that have a key role in a normal and pathological tissue remodelling. The whole group can be divided into subclasses, such as collagenases, gelatinases, stromelysins, and membrane type MMPs. ${ }^{1}$ In rheumatoid arthritis (RA), MMPs are indicated to be involved in excessive degradation of joint tissue.

MMP-3 (stromelysin-1) can degrade various components of joint tissue and activate proMMPs. ${ }^{1}$ It has been suggested that MMP-3 is a marker of synovitis derived from the joint because it is produced by synovial fibroblasts of the rheumatoid joints and not by normal synovial cells ${ }^{1}$ and correlates with inflammatory markers in the systemic circulation such as $\mathrm{C}$ reactive protein (CRP). ${ }^{2}$ Other MMP subclasses such as gelatinases and collagenases are expressed by cells of the pannus-cartilage or bone junction and by infiltrating inflammatory cells such as neutrophils and macrophages. Increased levels of collagenases and gelatinases are found not only at tissue level ${ }^{3}$ but also in the synovial fluid $(\mathrm{SF})^{4}$ and in the systemic circulation. ${ }^{1}$ Moreover, it has been indicated that high tissue levels of MMP-2 and -9 and systemic levels of MMP- 1 in patients with RA are associated with the development of joint erosions. ${ }^{56}$
The above mentioned studies show that significant progress has been made on identification of MMPs involved in the disease process in RA. However, studies on multiple MMP subclasses and/or the relationship between local and systemic levels of the different MMP subclasses are scarce.

This study was designed to provide a broad analysis of the MMPs which play a part in RA pathology and to investigate the relation between local and systemic levels of MMPs and their tissue inhibitor (TIMP). Furthermore, the relation between the studied MMPs and systemic marker of inflammation, CRP, and joint collagen degradation product, hydroxyproline (OHPro), in patients with RA was investigated.

\section{MATERIALS AND METHODS}

Hydroxyproline measurements

OHPro was measured in diluted SF samples by high performance liquid chromatography after acid hydrolysis, according to the method described by Bank et al. ${ }^{7}$

\section{ProMMPs and TIMP- 1}

ProMMP-3 and -13 and TIMP-1 were measured using the sandwich enzyme linked immunosorbent assay (ELISA; Amersham Biosciences, Little Chalfont, UK). ProMMP-1, -2, -8 , and -9 were detected using specific MMP activity assays (Biotrak activity assay, Amersham Biosciences, Little Chalfont, UK).$^{8}$

\section{MMP activity in MMP/ $\alpha_{2}$-macroglobulin complexes}

MMP activity in $\alpha_{2}$-macroglobulin $\left(\alpha_{2} M\right)$ complexes was determined using small fluorogenic substrates, according to the modified method described by Beekman et al, ${ }^{9}$ Riley et al, ${ }^{10}$ and DeGroot et al. ${ }^{11}$

\section{Paired serum/SF samples}

Paired SF and serum samples from 15 patients with RA and 13 with OA were collected during therapeutic arthrocentesis of a knee joint. Nine SF samples of traffic casualties, obtained at post mortem, were used as controls (material was obtained with the informed consent of relatives); 15 healthy volunteers provided control serum samples. Table 1 shows the basic characteristics of the patient groups.

\section{Statistical analysis}

The Kruskal-Wallis test was used to assess differences between study groups, which were further analysed using the Mann-Whitney U test. Correlations were evaluated using Pearson's correlation or Spearman rank correlation coefficients with SPSS software (Chicago, IL, USA). A value of $\mathrm{p} \leqslant 0.05$ was considered significant.

Abbreviations: $\alpha_{2} M, \alpha_{2}$-macroglobulin; CRP, $C$ reactive protein; MMPs, matrix metalloproteinases; OHPro, hydroxyproline; RA, rheumatoid arthritis; SF, synovial fluid; TIMP, tissue inhibitors of metalloproteinases. 
Table 1 Characteristics of the patients groups

\begin{tabular}{llll}
\hline Characteristic & RA & OA & Control serum \\
\hline Age (years) & $53(13.7)$ & $76.8(10)$ & $47.5(11.9)$ \\
Male/female & $3 / 12$ & $3 / 8$ & $9 / 6$ \\
Disease duration (years) & $6.7(6.3)$ & $5.2(2.5)$ & \\
CRP (mg/l) & $28.9(23.7)$ & $3(3.4)$ & \\
RF positive & 11 of 15 & & \\
\hline
\end{tabular}

Results are shown as mean (SD)

$C R P, C$ reactive protein; $R F$, rheumatoid factor.

\section{RESULTS}

Table 2 shows the median values of the SF and serum levels of the studied MMPs and TIMP-1. As expected, highly increased levels of MMPs were found in the SF of patients with RA as compared with the control group. Also, OA SF MMP levels were significantly higher than in the control group, although they were lower than in RA. In contrast with $\mathrm{SF}$, only proMMP-3, -8 , and -9 levels were increased in the serum of patients with RA when compared with the control group or patients with OA. ProMMP-13 levels were below the detection limit in both compartments in all groups.

Significant correlations were found between SF and serum proMMP-9 $\left(r_{\mathrm{s}}=0.718, \mathrm{p}<0.003\right)$ and proMMP-8 $\left(r_{\mathrm{s}}=0.664\right.$, $\mathrm{p}<0.007)$ levels in patients with RA. Further, levels of the studied MMPs were higher in SF than in the systemic circulation $(\mathrm{p}<0.001)$ in the RA group, except for proMMP-9 levels $(p=0.78)$.

To assess the degree of the continuing joint tissue degradation during acute joint effusion, OHPro levels in SF were analysed. No significant difference was found between the OHPro levels in RA and OA SF (median (25th-75th centiles): $14.4(10.8-17.2) \vee 11.8(9.3-19.8), p=0.68)$. A significant correlation was found between SF proMMP-9 levels and OHPro in the RA group (table 3). Also, a trend towards a correlation was seen between SF proMMP-8 and OHPro, whereas no correlation was found between OHPro and SF proMMP-3 or systemic CRP levels.

In $\mathrm{SF}$, the following distribution pattern of the proMMPs was seen: RA $>0 A>$ controls. However, TIMP-1 showed a different distribution pattern: $\mathrm{RA}=\mathrm{OA}>$ controls, which implies an imbalance within the proteolytic system in favour of the MMPs. Moreover, higher levels of MMP in complex
Table 3 Correlations, $r_{\mathrm{s}}(\mathrm{p})$ : SF MMPs $v$ SF OHPro and systemic CRP in RA

\begin{tabular}{lll}
\hline & OHPro & CRP \\
\hline MMP-3 & $0.305(0.27)$ & $0.696(0.004)^{\star}$ \\
MMP-8 & $0.49(0.06)$ & $0.196(0.48)$ \\
MMP-9 & $0.59(0.02)^{\star}$ & $0.147(0.602)$ \\
CRP & $0.116(0.68)$ & \\
\hline
\end{tabular}

*MMP-3: Pearson's correlation; MMP-8 and -9: Spearman's rank correlation test.

Correlations between synovial fluid (SF) proMMP-3, -8, and -9 levels, OHPro levels in SF, and systemic levels of CRP.

with $\alpha_{2}$ M (activated, but not-TIMP inhibited MMPs are likely to form complexes with $\alpha_{2} \mathrm{M}^{12}$ ) were found in patients with RA than in control or OA groups, strengthening the hypothesis of an MMP/TIMP imbalance.

\section{DISCUSSION}

This study provides an extensive analysis of MMPs, TIMP-1, and the MMP $/ \alpha_{2} M$ complex in paired serum and SF samples of patients with RA and OA and controls with healthy knees.

The highest MMP levels in the SF of patients with RA were found for proMMP-1, $-3,-8$, and -9 . Whereas MMP-3 and -9 were also detectable in control SF, MMP-1 and -8 were found in control SF only at extremely low concentrations. MMP levels in the SF of patients with OA were lower than in RA, but significantly higher than in control SF, indicating that use of OA material as a control for patients with RA may not always be appropriate. Interestingly, MMP-8 and MMP-9 levels correlated with each other in the SF of both RA and OA groups. Also, MMP-1 and MMP-3 levels correlated with each other in RA and OA groups, probably indicating the predominant cell source of these MMPs: macrophages and neutrophils for MMP-8 and -9 and synovial cells for MMP-1 and $-3 .{ }^{4}$ MMP analysis in the systemic circulation of patients with RA and OA indicated that not all MMP locally involved (as shown by the increased SF levels) are also increased in serum. High serum levels of MMP-3, -8 , and -9 were found in RA, whereas only MMP-8 and -9 were also found to be increased in the serum of patients with OA.

Furthermore, strong correlations between serum and SF levels of proMMP-8 and -9 , which were both increased in the SF and serum of patients with RA were found, suggesting

Table 2 Median (25th-75th centiles) values of serum and SF MMP levels

\begin{tabular}{|c|c|c|c|}
\hline & Controls & OA & RA \\
\hline \multicolumn{4}{|l|}{ Synovial fluid } \\
\hline MMP-1 (U/ml) & $0.0(0.0-0.01)$ & $0.28(0.21-1.23)^{\star *}$ & $5.9(3.6-11.3)^{*} \ddagger$ \\
\hline MMP-8 (U/ml) & $0.0(0.0-0.01)$ & $0.75(0.15-2.2)^{* *}$ & $10(4.7-30.0)^{*} \ddagger$ \\
\hline MMP-3 (ng/ml) & 177 (149-249) & $869(625-7401)^{* *}$ & $22044(14700-25800)^{*} \ddagger$ \\
\hline MMP-2 (U/ml) & $17(13-19)$ & $30(25-35)^{* *}$ & $31(30-34) \ddagger$ \\
\hline MMP-9 (U/ml) & $0.1(0.07-0.18)$ & $0.7(0.4-1.7)^{\star \star}$ & $4.0(2.3-6.3)^{*} \ddagger$ \\
\hline MMP-13 & Not detectable & Not detectable & Not detectable \\
\hline $\mathrm{MMP} / \alpha_{2} \mathrm{M}(\mathrm{U} / \mathrm{ml})$ & $37(34-41)$ & $55(43-124)^{\star *}$ & $470(334-1321)^{*} \ddagger$ \\
\hline TIMP-1 (ng/ml) & $322(0-1478)$ & 8961 (5287-16252)ฯ & 5717 (1951-9666)‡ \\
\hline \multicolumn{4}{|l|}{ Serum } \\
\hline MMP-1 (U/ml) & $0.06(0.02-0.1)$ & $0.06(0-0.1)$ & $0.06(0.06-0.1)$ \\
\hline MMP-8 (U/ml) & $0.64(0.6-1)$ & $1.12(0.7-2.6)^{9}$ & $4.4(1.0-6.6) \dagger \ddagger$ \\
\hline MMP-3 (ng/ml) & $16(11-25)$ & $17(12-28)$ & $85(36-117)^{\star} \ddagger$ \\
\hline MMP-2 (U/ml) & $5.6(4.9-6.4)$ & $6(4.9-7.0)$ & $6.15(5.6-8.0)$ \\
\hline MMP-9 (U/ml) & $2.1(1.6-2.6)$ & $3.0(2.0-3.6)$ & $4.6(3.0-6.4) \dagger \ddagger$ \\
\hline MMP-13 & Not detectable & Not detectable & Not detectable \\
\hline$M M P / \alpha_{2} M(U / m l)$ & $86(77-128)$ & $88(80-145)$ & $172(71-198) \S$ \\
\hline TIMP-1 (ng/ml) & $445(428-627)$ & $495(412-1368)$ & 345 (266-1088)† \\
\hline
\end{tabular}


that serum levels of these enzymes may be used to assess the situation in inflamed joints.

To elucidate the involvement of different MMP subclasses in joint inflammation and tissue degradation, MMP levels were compared with markers of systemic inflammation (CRP) and the collagen degradation marker, OHPro. Only proMMP-3 levels in SF correlated with CRP levels, which is consistent with findings by others. ${ }^{6}$ SF OHPro levels did not correlate with proMMP-3, whereas a significant correlation with proMMP-9 levels and a trend towards correlation with proMMP-8 were found. These results are in line with in vitro data on collagen degradation: collagen, one of the main components of the articular cartilage, can be specifically cleaved by collagenases into the characteristic $3 / 4$ and $1 / 4$ fragments, ${ }^{13}$ which can be further degraded by gelatinases. ${ }^{1}$ Thus, the results provide in vivo indication of MMP-9 involvement in collagen degradation in the joints of patients with RA. Taken together, these results show that MMP- 8 and -9 are likely to play a part in the degradation of the collagenous network of joint tissue, whereas proMMP-3 levels are likely to reflect the inflammatory component of the disease process.

An ample body of evidence suggests the presence of an MMP/TIMP imbalance in arthritic diseases. ${ }^{414}$ Based on molar ratios, TIMPs levels seem to be insufficient to counteract increased MMP production levels in RA. Our results of equally increased TIMP-1 levels in the serum and SF of patients with OA and RA and higher levels of proMMPs in RA are in line with this hypothesis. Furthermore, in the SF and serum of patients with RA high levels of active MMPs in complex with $\alpha_{2} \mathrm{M}$ are present, also indicating an MMP/TIMP imbalance in RA as compared with the normal situation or patients with OA. The importance of the MMP/TIMP imbalance in RA is also indicated by higher MMP activity levels at the inflammation site (SF) as compared with the serum levels, because no difference was seen between local and systemic levels in OA.

In conclusion, this study provides an extensive analysis of systemic and SF levels of major MMP subclasses in patients with RA and OA. Furthermore, the results $(a)$ show that systemic levels of MMP-8 and -9 are representative for the levels of these enzymes in the inflamed joints; $(b)$ may indicate involvement of MMP-9 in generation of collagen degradation products in the SF of patients with RA; and (c) are in accordance with the hypothesis of an MMP/TIMP imbalance in RA.

\section{Authors' affiliations}

I Tchetverikov, B van El, G H Kiers, N Verziil, J M TeKoppele, J DeGroot, R Hanemaaijer, Gaubius Laboratory, TNO Prevention and Health, Leiden, The Netherlands
I Tchetverikov, T W J Huizinga, Department of Rheumatology, Leiden University Medical Centre, Leiden, The Netherlands

H K Ronday, Department of Rheumatology, Leyenburg Hospital, The Hague, The Netherlands

Correspondence to: Dr R Hanemaaijer, TNO Prevention and Health, PO Box 2215, 2301 CE, Leiden, The Netherlands; R.Hanemaaijer@ pg.tno.nl

Accepted 17 August 2003

\section{REFERENCES}

1 Murphy G, Knauper V, Atkinson S, Butler G, English W, Hutton M, et al. Matrix metalloproteinases in arthritic disease. Arthritis Res 2002;4(suppl 3):S39-49

2 Posthumus MD, Limburg PC, Westra J, van Leeuwen MA, van Rijswijk MH. Serum matrix metalloproteinase 3 in early rheumatoid arthritis is correlated with disease activity and radiological progression. J Rheumatol 2000;27:2761-8

3 Konttinen YT, Ainola M, Valleala H, Ma J, Ida H, Mandelin J, et al. Analysis of 16 different matrix metalloproteinases (MMP-1 to MMP-20) in the synovial membrane: different profiles in trauma and rheumatoid arthritis. Ann Rheum Dis 1999;58:691-7.

4 Yoshihara Y, Nakamura H, Obata K, Yamada H, Hayakawa T, Fujikawa K, et al. Matrix metalloproteinases and tissue inhibitors of metalloproteinases in synovial fluids from patients with rheumatoid arthritis or osteoarthritis. Ann Rheum Dis 2000;59:455-61

5 Goldbach-Mansky R, Lee JM, Hoxworth JM, Smith D, Duray P, Schumacher RH Jr, et al. Active synovial matrix metalloproteinase-2 is associated with radiographic erosions in patients with early synovitis. Arthritis Res 2000;2:145-53.

6 Cunnane G, Fitzgerald O, Beeton C, Cawston TE, Bresnihan B. Early joint erosions and serum levels of matrix metalloproteinase 1, matrix metalloproteinase 3, and tissue inhibitor of metalloproteinases 1 in rheumatoid arthritis. Arthritis Rheum 2001;44:2263-74.

7 Bank RA, Krikken M, Beekman B, Stoop R, Maroudas A, Lafeber FP, et al. A simplified measurement of degraded collagen in tissues: application in healthy, fibrillated and osteoarthritic cartilage. Matrix Biol 1997;16:233-43.

8 Hanemaaijer R, Visser H, Konttinen YT, Koolwijk P, Verheijen JH. A novel and simple immunocapture assay for determination of gelatinase-B (MMP-9) activities in biological fluids: saliva from patients with Siögren's syndrome contain increased latent and active gelatinase-B levels. Matrix Biol 1998; 17:657-65.

9 Beekman B, Driifhout JW, Ronday HK, TeKoppele JM. Fluorogenic MMP activity assay for plasma including MMPs complexed to alpha 2 macroglobulin. Ann N Y Acad Sci 1999:878:150-8.

10 Riley GP, Curry V, DeGroot J, van El B, Verzijl N, Hazleman BL, et al. Matrix metalloproteinase activities and their relationship with collagen remodelling in tendon pathology. Matrix Biol 2002;21:185-95.

11 DeGroot J, Verziil N, Budde M, Biilsma JW, Lafeber FP, TeKoppele JM. Accumulation of advanced glycation end products decreases collagen turnover by bovine chondrocytes. Exp Cell Res 2001;266:303-10.

12 Cawston TE, Mercer E. Preferential binding of collagenase to alpha 2macroglobulin in the presence of the tissue inhibitor of metalloproteinases. FEBS Lett 1986;209:9-12.

13 Marini S, Fasciglione GF, De Sanctis G, D'Alessio S, Politi V, Coletta M. Cleavage of bovine collagen I by neutrophil collagenase MMP-8. Effect of $\mathrm{pH}$ on the catalytic properties as compared to synthetic substrates. J Biol Chem 2000;275: 18657-63.

14 Martel-Pelletier J, McCollum R, Fujimoto N, Obata K, Cloutier JM, Pelletier JP. Excess of metalloproteases over tissue inhibitor of metalloprotease may contribute to cartilage degradation in osteoarthritis and rheumatoid arthritis. Lab Invest 1994;70:807-15. 